\title{
Freezing of capillary waves at the glass transition
}

\author{
T. Seydel, ${ }^{1, *}$ M. Tolan, ${ }^{2}$ B. M. Ocko, ${ }^{3}$ O. H. Seeck, ${ }^{4}$ R. Weber, ${ }^{5}$ E. DiMasi, ${ }^{3}$ and W. Press ${ }^{5}$ \\ ${ }^{1}$ Institut Laue-Langevin, BP 156, F-38042 Grenoble, France \\ ${ }^{2}$ Experimentelle Physik 1, Universität Dortmund, Otto-Hahn-Strasse 4, D-44221 Dortmund, Germany \\ ${ }^{3}$ Physics Department, Brookhaven National Laboratory, Upton, New York 11973-5000 \\ ${ }^{4}$ IFF, FZ Jülich GmbH, D-52425 Jülich, Germany \\ 5 Institut für Experimentelle und Angewandte Physik, Christian-Albrechts-Universität, Leibnizstraße 17-19, D-24098 Kiel, Germany
}

(Received 4 May 2001; revised manuscript received 26 February 2002; published 13 May 2002)

\begin{abstract}
The freezing of capillary waves on glycerol surfaces is studied by in situ x-ray reflectivity measurements. A wide temperature range around the calorimetric glass transition temperature at $T_{g} \approx 186 \mathrm{~K}$ is investigated. For $T>250 \mathrm{~K}$ the obtained surface roughness as a function of the temperature differs significantly from the value predicted by the classical capillary waves theory. Below the temperature $T \approx 250 \mathrm{~K}$ the magnitude of the roughness remains constant. Furthermore, a large hysteresis, i.e., a large difference of the roughnesses measured during cooling and heating of the sample, is observed. These findings are discussed in terms of viscosity effects.
\end{abstract}

DOI: 10.1103/PhysRevB.65.184207～PACS number(s): 61.41.+e, 68.03.Kn, 68.15.+e, 61.10.-i

\section{INTRODUCTION}

While the microscopic fluctuations of liquid surfaces have been extensively investigated, very little is known about the surface of supercooled liquids or glasses and their glass transition. Since static structures of the bulk liquid and the glassy state are almost indistinguishable, $\mathrm{x}$-ray scattering experiments are virtually insensitive to the bulk glass transition. This is different at the surface, where the x-ray scattering contrast between the liquid and the vapor is large. It is well known that the surface of a liquid is roughened by thermally activated capillary waves. ${ }^{1-3}$ These surface waves lead to quasi-long-range correlations in the surface height which give rise to distinct features such as power-law tails in the diffuse scattering and a resolution dependent rms roughness. $^{4-8}$

In this paper we report in situ studies of the roughness of glycerol surfaces induced by capillary waves while the bulk liquid is supercooled and undergoes a glass transition. Glycerol was used because it is a prototypical glass at sufficiently low temperatures and the bulk properties of this material were extensively investigated in the past. ${ }^{9-12}$ The calorimetric glass transition temperature of glycerol is at $T_{g}=186 \mathrm{~K},{ }^{13}$ which is lower than the bulk melting transition $T_{m}=291 \mathrm{~K}$. Below $T_{m}$ noncrystalline glycerol may be considered as a supercooled liquid. Although it can be easily supercooled to $T \approx 235 \mathrm{~K}$, below this temperature glycerol crystallizes when slowly cooled. At sufficiently fast slew rates-rates of approximately $1.5 \mathrm{~K} / \mathrm{min}$-crystallization is circumvented. At $T \approx 200 \mathrm{~K}$ and for lower temperatures supercooled glycerol remains stable on experimentally accessible time scales. ${ }^{9,10}$

In the supercooled regime many capillary wave modes are overdamped due to the large viscosity. ${ }^{1,14}$ Recently such overdamped surface waves have been directly measured by $\mathrm{x}$-ray photon correlation spectroscopy (XPCS). ${ }^{15}$ Due to the surface sensitivity of these experiments the waves were unambiguously detected in the temperature range of $238 \mathrm{~K}$ $<T<273 \mathrm{~K}$ on lateral length scales from 100 down to
$5 \mu \mathrm{m}$. However, it is quite difficult to extend surfacesensitive XPCS experiments down to nanometer scales because even modern third-generation synchrotron sources are not able to deliver enough coherent x-ray photons for such experiments. Therefore, we have performed x-ray reflectivity measurements in order to determine the surface roughness $\sigma$ as a function of the temperature $T$ of the sample. Because $\sigma$ is related to the integral over all lateral wave modes accessible in an x-ray experiment, this type of measurement may be indirectly sensitive to nanometer length scales.

Since a glass is not in thermal equilibrium, its state depends on the thermal history. In addition, close to $T_{g}$ the experimental time scale crosses the time scale of the fluctuations since the viscosity is drastically enhanced and slows down all motion exponentially. It is not quite yet clear how the crossing of time scales and the damping of fluctuations by the large viscosity alters the surface roughness of a liquid when measured by $\mathrm{x}$-ray reflectivity. As we shall see later, our data from glycerol surfaces provide a picture which shows strong deviations from the hydrodynamic capillary waves theory. In addition, departures from a simple error function shaped electron density profile of the liquid/vapor interface are found for elevated temperatures.

In Sec. II the experimental details are described. This is followed by a section about $\mathrm{x}$-ray reflectivity from a liquid surface. The reflectivity measurements from glycerol surfaces are presented in Sec. IV. In Sec. V an attempt is made to explain the data in terms of different experimental time scales induced by the viscosity. A summary and conclusions are given in Sec. VI.

\section{EXPERIMENT}

The X-ray measurements were performed at the Harvard/ BNL liquid surface spectrometer at the NSLS beamline $\mathrm{X} 22 \mathrm{~B}$. The data were taken during two separate runs using wavelengths of $\lambda=1.24$ and $1.53 \AA$, respectively. The cor- 


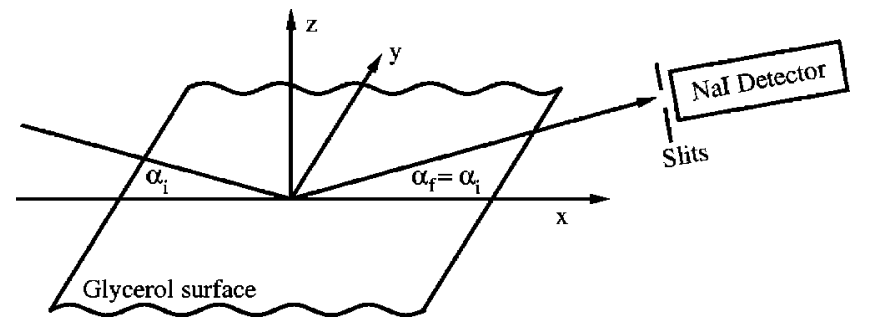

FIG. 1. Sketch of the experimental setup indicating the coordinate system with respect to the horizontal sample surface. The slits at the $\mathrm{NaI}$ detector define the experimental resolution. Footprint and guard slits are not shown.

responding angular resolutions $\Delta \alpha_{f}$ calculated from the vertical detector slit setting were 2.60 and $3.03 \mathrm{mrad}$ for the two configurations. A sketch of the setup is given in Fig. 1. Macroscopic glycerol films [material purchased from Fluka \# 49770 , purity better than $99.5 \%$ (Ref. 16)] of approximately $4.5-\mathrm{mm}$ thickness were prepared in a trough of $140-\mathrm{mm}$ diameter inside a sample chamber. ${ }^{17}$ The large diameter of the sample ensured that the illuminated surface was not affected by a meniscus of the liquid at the borders of the sample container. After filling the trough with glycerol, the sample chamber was evacuated to approximately 10 mbar in order to reduce the background scattering from the gas, and then hermetically sealed. The remaining pressure above the sample is caused by the vapor pressure of the glycerol at the specific sample preparation temperature. This may yield different levels of the measured background signal, since it is difficult to control the remaining pressure, i.e., to prepare samples under exactly the same starting conditions. The background level is a crucial point in x-ray reflectivity measurements from bulk liquid surfaces, since isotropic bulk scattering dominates the scattering for large wave-vector transfers. ${ }^{8}$ Thus the surface signal has to be determined by subtracting the separately measured bulk scattering background. Every source of additional background counts renders the precise determination of the surface signal more difficult by decreasing the surface signal to background ratio.

A vacuum $\left(p<10^{-5}\right.$ mbar) was maintained in an outer aluminum cell surrounding the sample chamber for thermal insulation. In order to check the homogeneity of the temperature distribution and to protocol the temperature inside the sample cell, two platinum resistor (Pt100) sensors which were coated by ceramics were dipped into the glycerol approximately $100 \mathrm{~mm}$ apart from each other. The two temperatures were in good agreement during all measurements. The x-ray beam penetrated both the inner and outer cell walls through Kapton foil windows. The cooling of the sample was accomplished with a constant flow of liquid nitrogen evaporating in a heat exchanger underneath the sample chamber. The flow rate was set by a control valve in the exhaust gas stream. The heating of the sample was done with an electric resistor connected to a Lakeshore temperature controller. A temperature stability of better than $0.02 \mathrm{~K}$ was achieved while a temperature range from 170 to $340 \mathrm{~K}$ was covered.

\section{X-RAY REFLECTIVITY FROM LIQUID SURFACES}

$\mathrm{X}$-ray reflectivity is a common tool to determine the density profile of a sample. In the case of a single surface of a bulk liquid this profile may be described by a single parameter, namely, the temperature-dependent width $\sigma(T)$ of the vapor/liquid interface. The measured reflectivity $R\left(q_{z}\right)$ is given by

$$
R\left(q_{z}\right)=\frac{1}{\sqrt{\pi}} \Gamma\left(\frac{1}{2}-\frac{k_{B} T}{4 \pi \gamma(T)} q_{z}^{2}\right) R_{F}\left(q_{z}\right) \exp \left\{-\sigma^{2}(T) q_{z}^{2}\right\}
$$

where $R_{F}\left(q_{z}\right)$ denotes the Fresnel reflectivity of an ideally smooth surface; $q_{z}=(4 \pi / \lambda) \sin \alpha_{i}$ is the vertical wave-vector transfer with $\alpha_{i}$ the incident angle of the radiation with respect to the surface, $\gamma(T)$ is the temperature-dependent surface tension, and $\Gamma(x)$ the gamma function. The gamma function only deviates from $\sqrt{\pi}$ for large $q_{z}$.

The usual expression for the measured total width of an interface broadened by capillary wave fluctuations is given by $^{4-6}$

$$
\sigma^{2}(T)=\sigma_{0}^{2}+\frac{k_{B} T}{2 \pi \gamma(T)} \ln \left(\frac{q_{\mathrm{u}}}{q_{1}}\right) .
$$

It was shown recently that the surface tension also depends on the lateral wave-vector transfer. ${ }^{3,8}$ However, this effect is small, and any wave-vector dependence of $\gamma(T)$ will be neglected in the following. In Eq. (2) an upper lateral wavevector cutoff $q_{u}$ was introduced. The magnitude of this wave-vector is commonly assumed to be on the order of $\pi / r_{m}$ with the molecular radius $r_{m}$. The intrinsic roughness $\sigma_{0}$ in Eq. (2) denotes the width of the interface in the absence of any capillary wave fluctuations. It is also expected to be on the order of $r_{m}$. A possible weak temperature dependence of $r_{m}$ and $\sigma_{0}$ is neglected in further considerations. Furthermore, a lower cutoff $q_{l}=q_{z} \Delta \alpha_{f} / 2$ was introduced into Eq. (2), where $\Delta \alpha_{f}$ is the angular acceptance of the detector. ${ }^{6}$ The inverse of this cutoff defines the largest lateral distance which contributes to the observed width $\sigma$ of the interface. For bulk liquid surfaces this lower cutoff is essentially given by the resolution of the experimental setup since the propagation of capillary waves is only hindered by the gravitational potential. For thin liquid films this cutoff may be altered, since here the much stronger van der Waalsinteraction prevents long-wavelength fluctuations. ${ }^{7}$

We note that the total intensity of the capillary waves spectrum is given by $S(q)=\int_{-\infty}^{+\infty} S(q, \omega) d \omega$, where $S(q, \omega)$ is the dynamic surface structure factor. $S(q)$ is independent of the viscosity according to the hydrodynamic capillary waves theory. ${ }^{18}$ Equation (2) results from integrating the capillary waves spectrum over the experimental resolution volume,

$$
\sigma^{2}(T)=\int_{q_{\mathrm{res}}} \int_{\omega_{\mathrm{res}}} S(q, \omega) d \omega d q,
$$

where the integral over $q$ is in fact a double integral taken over $q_{x}$ and $q_{y}$. The frequency resolution $\omega_{\text {res }}$ in Eq. 3 is 


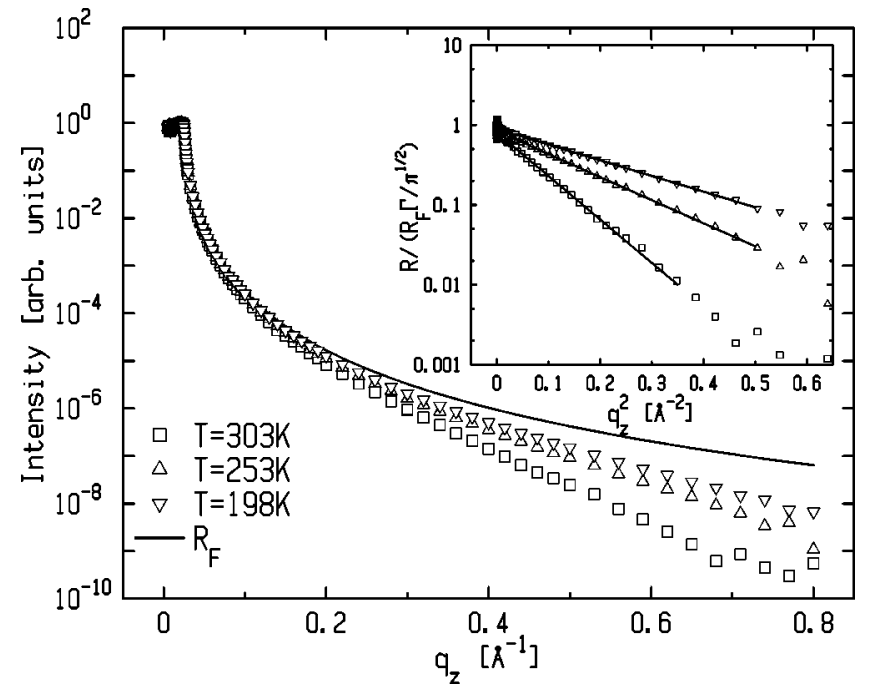

FIG. 2. Reflectivity data from glycerol surfaces after background subtraction and normalization (symbols) for three different temperatures $T$. The solid line is the calculated Fresnel reflectivity $R_{F}\left(q_{z}\right)$ for a sharp interface. The inset shows the data normalized by $\pi^{-1 / 2} \Gamma\left(\frac{1}{2}-\left[k_{B} T / 4 \pi \gamma(T)\right] q_{z}^{2}\right) R_{F}\left(q_{z}\right)$ on a logarithmic scale vs. $q_{z}^{2}$. The solid lines are linear fits where the slope determines the rms-roughness of the interface.

assumed to be infinite, and this assumption may no longer hold for very viscous liquids where the frequency spectrum of the capillary waves is altered. ${ }^{18}$

\section{X-RAY REFLECTIVITY MEASUREMENTS}

Examples of reflectivity data from glycerol surfaces at different temperatures after background subtraction and normalization are shown in Fig. 2. These data have been taken after waiting for several hours at each adjusted temperature. The background has been measured by performing the reflectivity scans with a constant $0.4^{\circ}$ angular offset of the detector out of the scattering plane (the $q_{y}$ direction). The symmetry of the background has been tested by performing detector scans around the scattering plane at constant incident and take-off angle. The background (essentially scattering from the bulk) is expected to be nearly independent of $q_{z}$ (except for the bulk liquid structure peak at very large $q_{z}$ ). It is subtracted from the measured reflectivity data to obtain what is called the "true reflectivity" from the liquid surface. $^{6,8}$ In the inset of Fig. 2 the intensity $R\left(q_{z}\right)$ normalized by

$$
\pi^{-1 / 2} \Gamma\left(\frac{1}{2}-\frac{k_{B} T}{4 \pi \gamma(T)} q_{z}^{2}\right) R_{F}\left(q_{z}\right)
$$

with the Fresnel reflectivity $R_{F}\left(q_{z}\right)$ plotted on a logarithmic scale vs $q_{z}^{2}$. According to Eq. (1) the slope of the fit to the data (solid lines) directly corresponds to the width $\sigma^{2}$ of the liquid/gas interface. The fit yields $\sigma=3.5 \AA$ for $T$ $=303 \mathrm{~K}, \sigma=2.6 \AA$ for $T=253 \mathrm{~K}$ and $\sigma=2.1 \AA$ for $T$ $=198 \mathrm{~K}$.
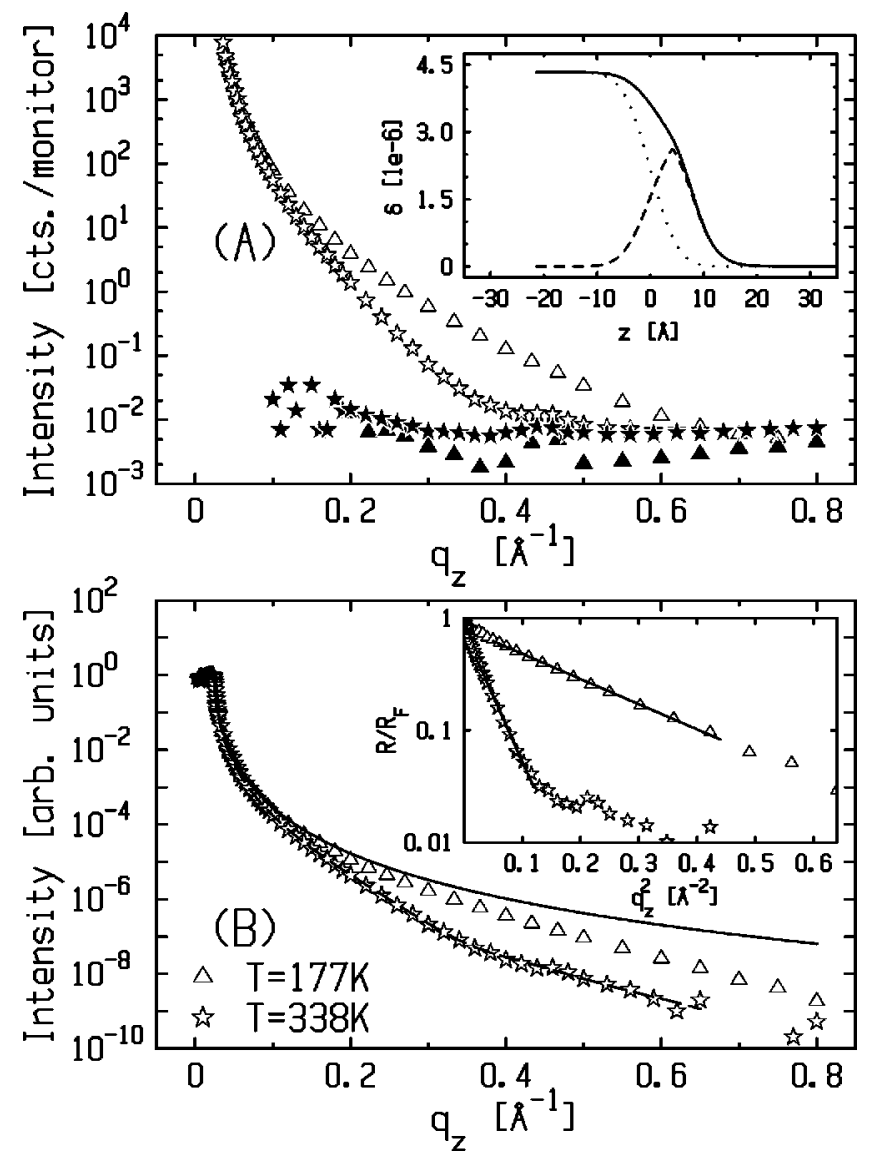

FIG. 3. (A) Reflectivity data (upper two curves, open symbols) for a low temperature $(T=177 \mathrm{~K})$ and a high temperature $(T$ $=338 \mathrm{~K})$, respectively, before background subtraction and the corresponding bulk scattering background scans (filled symbols). Both background scans show a slight $q_{z}$ dependence with a maximum at $q_{z} \sim 0.45 \AA^{-1}$. The overall bulk scattering background level rises with rising temperature. The inset shows the fitted real part $\delta$ of the refractive index profile (full line) perpendicular to the surface for the high-temperature data, assuming bulk glycerol (dotted line) covered by a thin layer (dashed line). (B) The reflectivity data from (A) after background subtraction and normalization of the maximum to unity. The solid line is the Fresnel reflectivity $R_{F}$ of an ideally smooth surface. The dashed line is calculated from the fit of a layer model to the high temperature data [see the inset of $(\mathrm{A})]$. The inset of (B) shows the data after normalization to $\pi^{-1 / 2} \Gamma\left(\frac{1}{2}\right.$ $\left.-\left[k_{B} T / 4 \pi \gamma(T)\right] q_{z}^{2}\right) R_{F}\left(q_{z}\right)$. A slight oscillation at large $q_{z}$ can be seen in the high-temperature case. The solid lines are linear fits.

However, it turns out that especially for elevated temperatures $(T>300 \mathrm{~K})$ the observed behavior deviates from the simple picture of scattering from a single surface. In Fig. $3(\mathrm{~A})$, the reflectivity and background scans for the temperatures $\mathrm{T}=177$ and $338 \mathrm{~K}$ are given. The observed background signal shows a structure at $q_{z} \sim 0.45 \AA^{-1}$ which affects the corresponding true specular reflectivity signal for $q_{z}$ $>0.5 \AA^{-1}$. This is shown in Fig. 3(B), where the reflectivity data after background subtraction are presented. For large $q_{z}$, deviations of the high- $T$ data from the theoretical reflectivity curves are obvious. This is highlighted in the inset of Fig. 3(B), where a straight line indicates scattering which- 
may be associated with capillary waves, as discussed in Sec. III. It can be seen that the data corresponding to $T=338 \mathrm{~K}$ show rather strong departures from a straight line. Such features may indicate the formation of a layer of different density at the glycerol surfaces. This indication, which would give rise to the observed slight oscillation or flattening of the curves for large $q_{z}$ values, is found only for the hightemperature data where condensation of material or segregation is unlikely. Figure 3(A) illustrates that the feature in the background is present for all temperatures. It shows up in the reflectivity data only for higher $T$, because here the roughness of the liquid surface is large and hence the reflected intensity from the surface is damped much more strongly than for the lower temperatures. We therefore attribute the observed structure to a long-range correlation with a length of approximately $20 \AA$ in the bulk due to the H-bond network of glycerol molecules. A peak corresponding to the structure in our background scans has been reported from neutron small-angle scattering experiments on bulk glycerol. ${ }^{19}$ We have observed that an augmentation of the temperature is always accompanied by an increase of the overall background level. We note that this difficulty may become even more severe for liquids with higher vapor pressure. In our example, the temperature of $T=338 \mathrm{~K}$ is an extreme case, where the vapor pressure of the glycerol is already significantly higher than that at room temperature.

The slight oscillation or flattening of the reflectivity at high $q_{z}$ values renders the precise determination of $\sigma^{2}$ difficult for higher temperatures and leads to larger error bars. The roughnesses derived from the fitted slopes according to Eq. (1) are $\sigma=2.4 \AA$ for $T=177 \mathrm{~K}$ and $\sigma=5.3 \AA$ for $T$ $=338 \mathrm{~K}$, where the fit range in $q_{z}$ has been limited to the region of sufficient signal-to-background ratio. In addition, it turns out that the gamma function has no significant effect on the determination of the roughness. The fit of a layer model to the $T=338 \mathrm{~K}$ data yields a single surface layer with a thickness of $7.9 \AA$ and a surface roughness of $4.0 \AA$. This fit therefore suggests a modified liquid/vapor interface which cannot be described by a single error function profile. According to this model, the electron density of the layer amounts to $72 \%$ of the bulk electron density. The fit result is given as an inset of Fig. 3(A), where the real part of the refractive index $\delta$ of the bulk glycerol (dotted line), the assumed surface layer (dashed line), and the sum of both (full line) are shown. The reflectivity calculated from this fit is given as dashed line in Fig. 3(B). The fit of the layer model to the low-temperature data in Fig. 3 converges to a surface layer of zero thickness and a bulk surface roughness of $2.4 \AA$, in agreement with the fit according to Eq. (1).

At very high $q_{z}$ values, the scattering arises almost entirely from the bulk, and the peak due to the nearest-neighbor correlations within the liquid becomes visible (see Fig. 4). From the linear relationship of the peak position with temperature the volume expansion coefficient is calculated to be $7.3 \times 10^{-4} / \mathrm{K}$ at $200 \mathrm{~K}$, in good agreement with the literature value. The bulk correlation length defined by $\xi=2 \pi / \mathrm{FWHM}$ ranges from $\xi=(49 \pm 2) \AA$ at $T=298 \mathrm{~K}$ to $\xi=(56 \pm 2) \AA$ at $T=201 \mathrm{~K}$. Therein FWHM denotes the full width at half maximum of the peak.

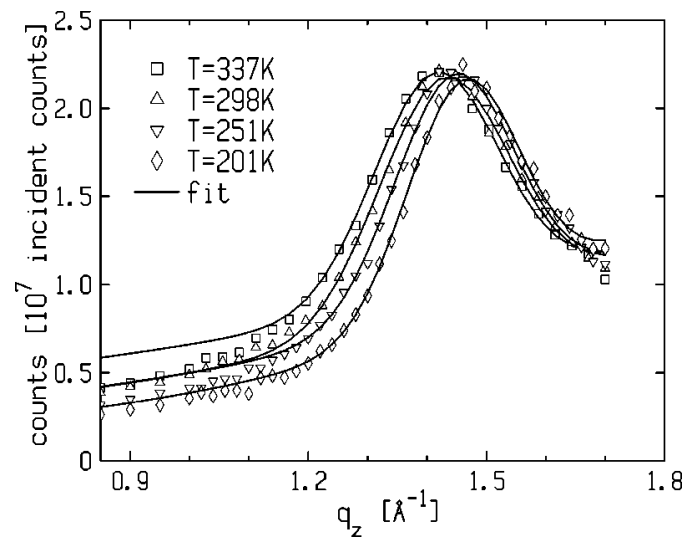

FIG. 4. Reflectivity of glycerol for different temperatures and large $q_{z}$. In this region the scattering stems essentially from the bulk and the peak is due to the nearest-neighbor correlation within the liquid. The lines are fits to a sum of a gaussian and background.

Figure 5 shows the roughness values $\sigma^{2}(T)$ (open squares) vs the temperature $T$ as obtained from full reflectivity scans. The corresponding reflectivity scans (see the inset) have been taken during a stepwise cooling of the same sample after waiting for several hours at each adjusted temperature and changing the illuminated spot on the sample to avoid beam damage. The time required for a complete reflectivity scan ranges from 20 to $30 \mathrm{~min}$. A decrease of the roughness with decreasing temperature for $T>250 \mathrm{~K}$ can be seen. One may suspect that the roughness remains constant at $\sigma \approx 2 \AA$ for temperatures below $250 \mathrm{~K}$. The dashed line in Fig. 5 represents the calculated roughness due to capillary

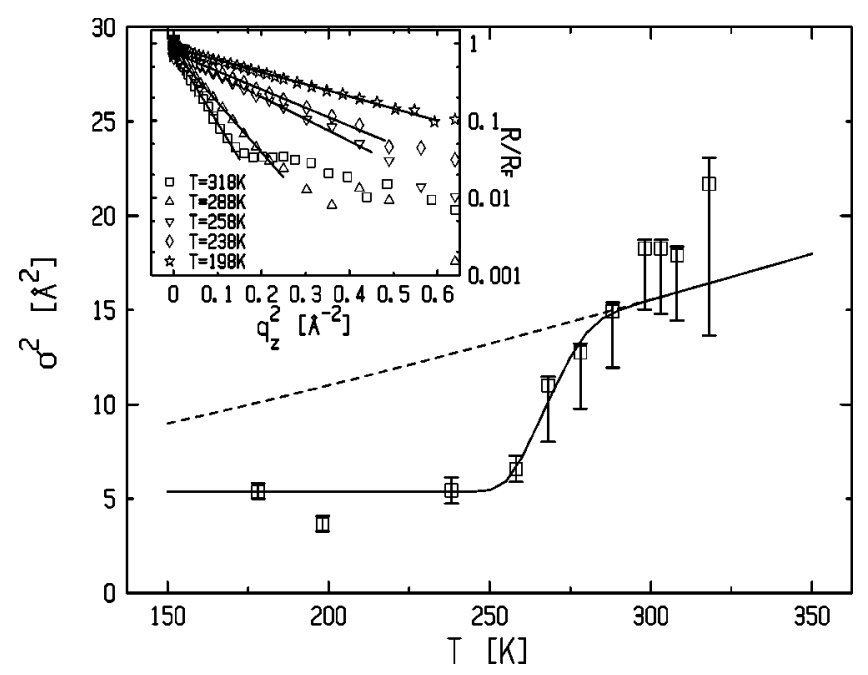

FIG. 5. Roughness $\sigma^{2}$ vs $T$ (symbols) obtained from complete reflectivity scans of a glycerol surface assuming the validity of Eq. (1). The solid line is a guide to the eye obtained by fitting a simplistic model where a finite frequency resolution of the experiment has been assumed. The upper dashed line shows the calculation for equilibrium capillary waves [Eq. (2)]. In the inset, some of the corresponding reflectivity scans normalized to $R_{F}$ are shown, the solid lines again being linear fits. The error bars on the fitted $\sigma^{2}$ take a possible layering at the surface into account, which would give rise to a lower rms roughness. 


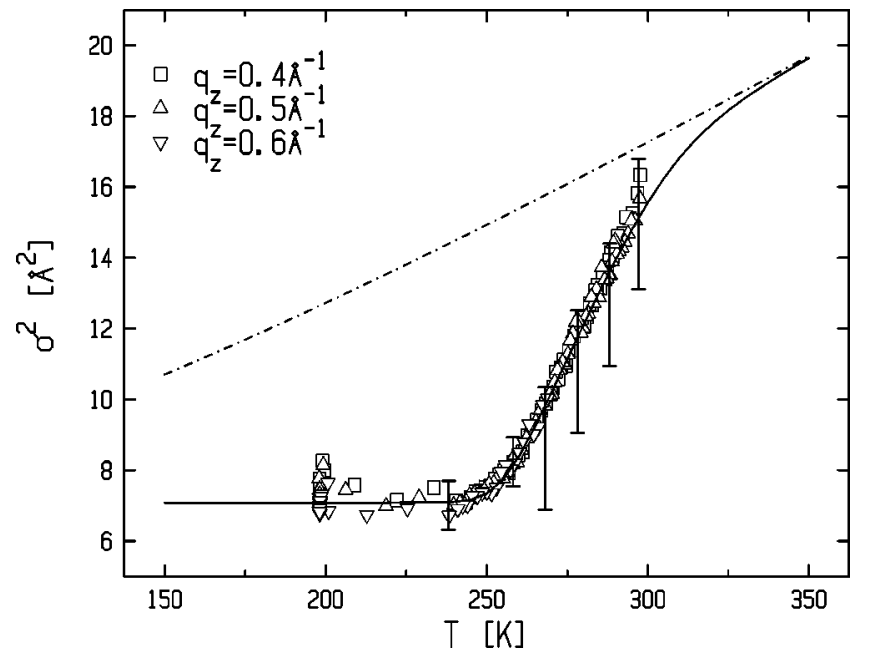

FIG. 6. Roughness $\sigma^{2}$ vs $T$ (symbols) obtained from reflectivity data at $q_{z}=0.4,0.5$, and $0.6 \AA^{-1}$ of a glycerol surface during a continuous cooling run assuming the validity of Eq. (1). The error bars are given analogously to Fig. 5, and take a possible layering at the surface into account. The solid line is a guide to the eye obtained by fitting a simplistic model where a finite frequency resolution of the experiment has been assumed. The upper dash-dotted line shows the calculation for equilibrium capillary waves [Eq. (2)].

waves as given by Eq. (2), where an experimentally fixed value for $q_{l}$ (Ref. 20) and an intrinsic width of $\sigma_{0} \approx r_{m}$ $\approx 2 \AA$ were used. The surface tension $\gamma(T)$ has been measured independently with the conventional Wilhelmy method. The temperature dependence of $\gamma(T)$ was found to be weak in comparison with the strong temperature dependence of the viscosity $\eta(T){ }^{21}$ At room temperature $\gamma(T)$ $\approx 0.05 \mathrm{~N} / \mathrm{m}$ was obtained.

We note that the symbols in Fig. 5 are the fitted values of $\sigma^{2}(T)$ assuming the validity of Eq. (1). The fit range in $q_{z}$ has always been set according to sufficient surface signal to background ratio. This is shown in the inset of Fig. 5, where the linear fits are only able to explain the high temperature data for $q_{z}^{2}<0.2 \AA^{-2}$. However, as stated above, a certain density profile due to layering at the surface may as well be considered. The error bars in Fig. 5 take this into account. While the upper limit for each data point is given rather accurately by the linear fits a possible layer at the surface would lead to a considerable reduction of $\sigma^{2}$. This is the reason why the error bars are very asymmetrical.

Since a system in the glassy state is not in thermal equilibrium one may expect that the measured results depend on the time over which the experiment averages and on the thermal history of the system as well. In our experiment the data acquisition can be done much faster, since Eq. (1) shows that the roughness of a liquid surface is already determined by the reflectivity at a single $q_{z}$ value. This allows a determination of $\sigma^{2}(T)$ during a continuous cooling of the sample, with the assumption of no surface layering effects.

The symbols in Fig. 6 show the $\sigma^{2}(T)$ data as obtained at three discrete $q_{z}$ values while the sample was continuously cooled with a slew rate of $0.2 \mathrm{~K} / \mathrm{min}$ for $T>240 \mathrm{~K}$ and 1.5 $\mathrm{K} / \mathrm{min}$ below. Data at $q_{z}=0.6 \AA^{-1}$ were only taken at low

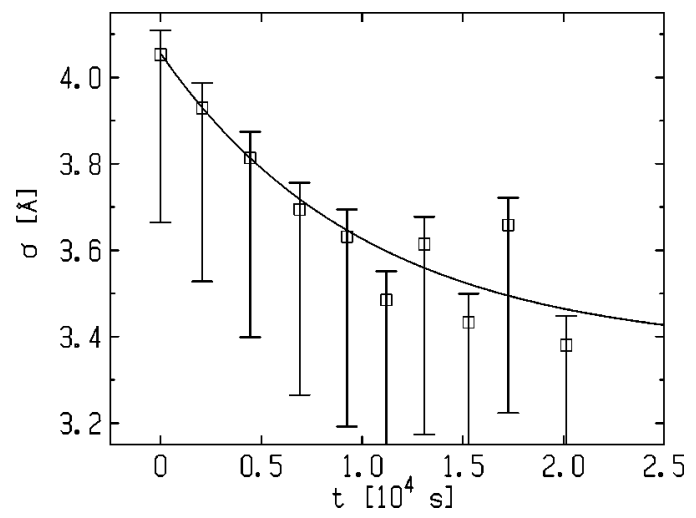

FIG. 7. Roughness $\sigma$ vs time (symbols) obtained from complete reflectivity scans of a glycerol surface as a function of time at a constant temperature $T=278 \mathrm{~K}$. The large error bars are based on the assumption of a possible systematic error due to a layering at the surface, and do not affect the overall trend. The solid line is a fit with a simple exponential form.

temperatures $T<270 \mathrm{~K}$, where the surface signal-tobackground ratio is sufficient. The temperature was kept constant at $198 \mathrm{~K}$ for several hours. To prevent beam damage, the illuminated spot on the sample was varied during the data collection. At $T=198 \mathrm{~K}$ the spot was not further changed. This may explain the slight increase of $\sigma^{2}$ at the final temperature. All $\sigma^{2}(T)$ values were computed with Eq. (1) using the same factor to normalize the intensity to total reflection. The roughness as determined from complete reflectivity scans did not change over several hours at $T=198 \mathrm{~K}$ and remained at the same value while the sample was cooled further to $T=188 \mathrm{~K}$ and finally to $T=178 \mathrm{~K}$ below the calorimetric glass transition temperature $T_{g}$. The data in Fig. 6 (open symbols) are in qualitative agreement with those of Fig. 5: Above $T=260 \mathrm{~K}$ an almost linear increase of $\sigma^{2}$ with $T$ is observed, below $T=240 \mathrm{~K}$ the roughness remains constant.

Figure 6 indicates that the glycerol surface already becomes static on a time scale of minutes at a temperature $T^{*} \approx 250 \mathrm{~K}$. This temperature is considerably larger than the calorimetric glass transition temperature $T_{g}=186 \mathrm{~K}$, where all microscopic motion in the bulk essentially stops. The slope of the $T>260 \mathrm{~K}$ data is approximately $0.2 \AA^{2} / \mathrm{K}$, while the usual capillary waves model [see Eq. (2) and the dash-dotted line in Fig. 6] yields the much smaller value of $0.04 \AA^{2} / K$. It is obvious that the measured rms-roughnesses are always smaller than the values predicted by Eq. (2).

In Fig. 7 the roughness calculated from reflectivity scans which have been taken consecutively at $T=278 \mathrm{~K}$ over a period of several hours is shown. Before reaching this temperature, the sample is cooled at a rate of $1.5 \mathrm{~K} / \mathrm{min}$. It can be seen that even after several hours the surface roughness still decreases. The crossing of the experimental time scale with that of the fluctuations is obvious.

We also found that hysteresis effects as a result of subsequent cooling and heating of the sample have a significant influence on the roughness. An example of such a measurement is shown in Fig. 8. The sample was prepared at $T$ $=320 \mathrm{~K}$, and cooled stepwise. At each temperature, a com- 


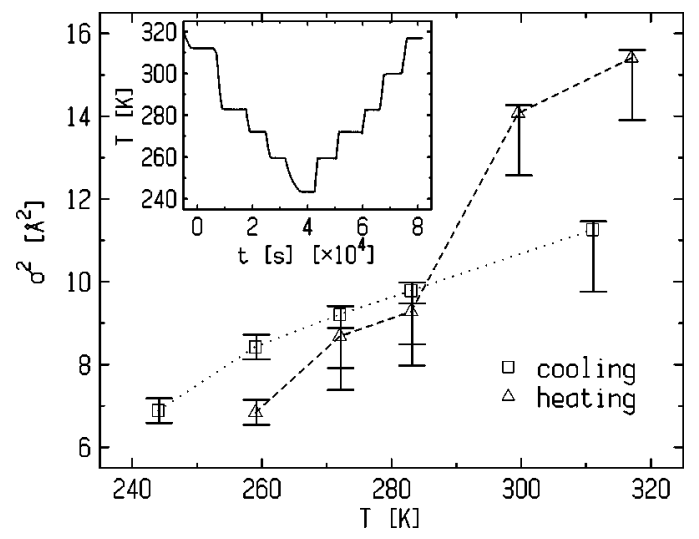

FIG. 8. Roughness values obtained from reflectivity scans during a stepwise cooling and re-heating of the same sample. The dashed lines are a guide to the eye. A strong hysteresis effect is obvious. The inset displays the temperature as a function of time during the experiment.

plete reflectivity scan was taken. The hysteresis effect hampers the exact reproducibility of the starting conditions for a measurement. It turned out that the shape of the hysteresis considerably depends on the thermal history of the samples.

\section{DISCUSSION}

Principally different time scales can be expected to be relevant for the interpretation of the data. The sample is slowly evolving while it is a (supercooled) liquid, rendering the experiment sensitive to the large time scales of (i) the thermal history of the sample, (ii) the integration time for each data point, and (iii) the time for the collection of a set of data. Since the frequency spectrum of the capillary waves is altered on a supercooled liquid and both very slow wave modes (overdamped capillary waves) and high-frequency modes (elastic shear waves) appear, ${ }^{18}$ the experiment may become sensitive to its frequency resolution and thus introduce a short time scale. The presence of these different time scales renders the data interpretation difficult.

The data shown in Figs. 5 and 6 are in disagreement with the roughness predicted by Eq. (2). Over a wide temperature range, the roughness values obtained from the reflectivity measurements are significantly smaller than expected according to the equilibrium capillary waves theory. The calculation according to Eq. (2), assuming the independently measured macroscopic surface tension, ${ }^{21}$ is indicated by dashed lines in the figures. As already mentioned, the deviation of the roughness from the magnitude expected by the equilibrium capillary waves theory is in qualitative agreement for the data shown in Fig. 5, i.e. after waiting for several hours at each temperature, and for the continously cooled sample (Fig. 6). In the continously cooled case, the minimum roughness at the low temperatures is slightly larger. At the high cooling rate the sample may be further away from equilibrium.

Our data cannot yet be understood quantitatively in terms of a capillary wave model based on the viscoelastic theory. We note that possible changes of motion and the viscosity at very short scales are subject to an ongoing debate. ${ }^{22-24}$ The contribution of overdamped capillary wave modes to the roughness was discussed by Jeng et al. ${ }^{25}$

Finally, we discuss the effect of a possible layering at the glycerol surface as observed in the data for large $q_{z}$ and elevated temperatures (see Fig. 3). Here it is surprising that the data show this effect for high temperatures above $275 \mathrm{~K}$, while the low-temperature data may be explained quite well with the assumption of scattering from a single surface with an error function like electron-density profile. A microscopic explanation for this layering or modified density profile which can be assumed to explain the slight oscillation or flattening of the $R / R_{F}$ data for $q_{z}>0.5 \AA^{-1}$ is not available yet. Temperature-dependent layering effects are already known from surfaces of long-chain liquid alkanes. ${ }^{26}$ However, as already stated in Sec. IV, the data shown in Fig. 3 suggest the following interpretation: The feature at $q_{z}$ $\approx 0.45 \AA^{-1}$ which leads to the slight oscillation in the $R / R_{F}$ curve is also visible in the background for all temperatures. Hence a characteristic bulk length scale of approximately $20 \AA$ may be attributed to this structure. For low temperatures the roughness of the surface is small, and hence this structure is orders of magnitude weaker than the reflected $\mathrm{X}$-ray signal [see Fig. 3(A)]. After background subtraction no additional feature shows up, suggesting that this structure is not present in the vertical density profiles. The situation changes for higher temperatures. Here the reflectivity drops faster due to the enhanced surface roughness. The background corrected reflectivity now also shows a feature at $q_{z}$ $\approx 0.45 \AA^{-1}$ which gives rise to a modulation, indicating a deviation from a simple error function profile at the surface. The net result may be that the feature stemming from the H-bond network shows up in the near-surface region, and hence leads to a modified density profile at the surface as disussed before. It is also possible that other yet unknown effects, e.g., due to the temperature dependence of the vapor pressure of the glycerol and to a possibly predominant orientation of the glycerol molecules at the surface might additionally, have to be taken into account.

\section{CONCLUSIONS}

In summary, we have presented an in situ study of the roughness of glycerol surfaces in a large temperature range while the bulk liquid is supercooled and undergoes a glass transition. We have shown that the capillary waves freeze on glycerol surfaces when the material is transformed to the supercooled state. This freezing occurs at much higher temperatures than the calorimetric glass transition temperature $T_{g}$ of the bulk material. It is also found that this does not necessarily mean that $T_{g}$ is altered at the surface, since capillary waves are quasi-long-ranged fluctuations in contrast to the short-ranged fluctuations in a liquid which extend on length scales on the order of the bulk correlation length. Furthermore, one has to take into account that the measurements become sensitive to experimental time scales and that the contribution of overdamped modes to the measured roughness is not yet well understood. In addition, the data for temperatures above $T=275 \mathrm{~K}$ indicate deviations of the 
electron density profile of the vapor/liquid interface from a simple error function, as would be expected for a surface roughness which is only given by capillary wave fluctuations. It is surprising that these differences, which may be attributed to a glycerol layer of reduced electron density, are found only for high temperatures. It appears that the viscosity becomes an important parameter in the determination of the capillary wave roughness on highly viscous liquids in addition to the surface tension by inducing different time scales to which the experiment becomes sensitive.

Our results may contribute to the fundamental understanding of overdamped and frozen-in capillary waves on viscous liquids. In addition, the understanding of x-ray scattering measurements from surfaces of other viscous liquids and polymers may benefit from our study.

\section{ACKNOWLEDGMENTS}

This work was supported by the "Deutsche Forschungsgemeinschaft" (Project Nos. Pr325/9-1,2,3 and Pr325/12-1). The measurements were carried out at beamline X22B at the National Synchrotron Light Source at Brookhaven National Laboratory, which is supported by the U.S. Department of Energy, Division of Materials Sciences and Division of Chemical Sciences under Contract No. DE-AC0298CH10886. We thank Henning Kraack (Bar-Ilan University, Israel) for the temperature-dependent macroscopic surface tension measurements on glycerol. Stimulating discussions with J. Jäckle (Universität Tübingen), S.K. Sinha, L. Lurio (Advanced Photon Source), and A. Madsen (European Synchrotron Radiation Facility) are gratefully acknowledged.
*Corresponding author. Electronic address: seydel@ill.fr

${ }^{1}$ V.G. Levich, Physicochemical Hydrodynamics (Prentice-Hall, Englewood Cliffs, NJ, 1962).

${ }^{2}$ F.P. Buff, R.A. Lovett, and F.H. Stillinger, Phys. Rev. Lett. 15, 621 (1965).

${ }^{3}$ K.R. Mecke and S. Dietrich, Phys. Rev. E 59, 6766 (1999).

${ }^{4}$ A. Braslau, P.S. Pershan, G. Swislow, B.M. Ocko, and J. AlsNielsen, Phys. Rev. A 38, 2457 (1988).

${ }^{5}$ M.K. Sanyal, S.K. Sinha, K.G. Huang, and B.M. Ocko, Phys. Rev. Lett. 66, 628 (1991).

${ }^{6}$ B.M. Ocko, X.Z. Wu, E.B. Sirota, S.K. Sinha, and M. Deutsch, Phys. Rev. Lett. 72, 242 (1994).

${ }^{7}$ A.K. Doerr, M. Tolan, W. Prange, J.-P. Schlomka, T. Seydel, W. Press, D. Smilgies, and B. Struth, Phys. Rev. Lett. 83, 3470 (1999).

${ }^{8}$ C. Fradin, A. Braslau, D. Luzet, D. Smilgies, M. Alba, M. Boudet, K. Mecke, and J. Daillant, Nature (London) 403, 871 (2000).

${ }^{9}$ J. Wuttke, W. Petry, G. Coddens, and F. Fujara, Phys. Rev. E 52, 4026 (1995).

${ }^{10}$ F.J. Bermejo, A. Criado, A. de Andres, E. Enisco, and H. Schober, Phys. Rev. B 53, 5259 (1996).

${ }^{11}$ P. Lunkenheimer, A. Pimenov, M. Dressel, Yu.G. Goncharov, R. Böhmer, and A. Loidl, Phys. Rev. Lett. 77, 318 (1996).

${ }^{12}$ F. Fujara, W. Petry, R.M. Diehl, W. Schnauss, and H. Sillescu, Europhys. Lett. 14, 563 (1991).

${ }^{13}$ M.R. Carpenter, D.B. Davies, and A.J. Matheson, J. Chem. Phys.
46, 2451 (1967).

${ }^{14}$ D. Byrne and J.C. Earnshaw, J. Phys. D 12, 1133 (1979).

${ }^{15}$ T. Seydel, A. Madsen, M. Tolan, G. Grübel, and W. Press, Phys. Rev. B 63, 073409 (2001).

${ }^{16}$ The main residual component in glycerol is water. Since water mixes with glycerol at any ratio and has a higher surface tension than glycerol, it does not affect the surface.

${ }^{17}$ T. Seydel, Ph.D. thesis, University of Kiel, Germany, 2000.

${ }^{18}$ J. Jäckle and K. Kawasaki, J. Phys.: Condens. Matter 7, 4351 (1995).

${ }^{19}$ D.C. Champeney and R.N. Joarder, Mol. Phys. 58, 337 (1986).

${ }^{20}$ The $q_{z}$ dependence of $q_{l}$ has been neglected by assuming a constant $q_{z}=0.4 \AA^{-1}$.

${ }^{21}$ The temperature-dependent measurements of the surface tension were done by Henning Kraack (Bar Ilan University, Israel). The surface tension $\gamma(T)$ changes about $20 \%$ within the temperature range of 280-330 K. We have extrapolated these data to lower temperatures assuming a $1 / T$ dependence.

${ }^{22}$ L.F. Phillips, Chem. Phys. Lett. 330, 15 (2000).

${ }^{23}$ G.J. Simpson and K.L. Rowlen, Chem. Phys. Lett. 309, 117 (1999).

${ }^{24}$ F. Brochard-Wyart and P.G. de Gennes, Europhys. J. E 1, 93 (2000).

${ }^{25}$ U-Ser Jeng, Levon Esibov, Lowell Crow, and Albert Steyerl, J. Phys. (Paris) 10, 4955 (1998).

${ }^{26}$ X.Z. Wu, E.B. Sirota, S.K. Sinha, B.M. Ocko, and M. Deutsch, Phys. Rev. Lett. 70, 958 (1993). 